\section{RESUME}

La protection des zones exposées à des risques d'éboulements rocheux nécessite la mise en œuvre de moyens complémentaires adaptés à chaque cas particulier.

L'article présente les principales mesures de protection et de surveillance mises en œuvre pour la protection d'un village des Alpes de Savoie à la suite d'un éboulement rocheux d'un millier de mètres cubes provenant d'une falaise instable.

\section{SUMMARY}

The protection of zones exposed to rock falls risks requires the carrying out of further means adapted to each particular case.

The paper describes the principal protection and monitoring measurements carried out for the safety of a village located in the "Alps of Savoien, due to rock falls of a thousand cubic metres coming from an unstable cliff. 


\title{
B. Protection des zones exposées à des éboulements rocheux
}

\author{
Contribution des méthodes de surveillance
}

par L. ROCHET

\section{INTRODUCTION}

La protection des zones exposées à des risques d'éboulement rocheux est un problème à la fois fréquent et difficile. Les éboulements peuvent être la conséquence plus ou moins proche ou éloignée de modifications apportées à l'équilibre naturel des masses rocheuses et ce doit être un souci permanent des ingénieurs que d'éviter de perturber dangereusement les équilibres naturels. Mais parallèlement, l'observation montre que ces phénomènes sont inscrits dans la nature des choses et font partie des mécanismes naturels d'évolution des reliefs. Leur manifestation présente un caractère inéluctable sans qu'il soit généralement possible d'en prévoir l'échéance. S'agissant de phénomènes à cinématique rapide les manifestations apparaissent souvent brutales et laissent un délai trop court pour permettre à ce stade la mise en cuvre de mesures de protection.
Dans ces conditions, la protection doit être recherchée dans deux directions :

- la mise en place de dispositifs de protection ou de renforcement préventifs ;

- la recherche et l'exploitation de critères objectifs susceptibles de constituer des signes avertisseurs suffisamment sensibles permettant de mettre en évidence toute aggravation dangereuse de l'instabilité.

Quels que soient les moyens mis en œuvre, l'appréciation des risques dans chaque cas particulier conserve un caractère de subjectivité que l'on ne peut totalement éliminer. De même, la protection ne peut être absolue et les moyens mis en œuvre résultent toujours d'un compromis entre ce qui serait souhaitable et ce qui est possible. Il importe au spécialiste d'apprécier où se situe la limite au-dessous de laquelle la protection devient insuffisante.

\section{EXEMPLE DE PROTECTION D'UNE ZONE HABITEE}

\subsection{Données générales}

Un éboulement d'un millier de mètres cubes environ provenant d'un éperon rocheux dominant le village de Saint-Jean (commune de La Perrière, Savoie) situé sur la RN 515 entre Moutiers et Bozel, s'est produit au début de l'hiver 1975. L'éboulement s'est propagé en créant une trouée dans la couverture forestière du versant jusqu'aux abords du village (fig. 1).

Dans cette zone, les terrains schisto-gréseux d'âge houiller déterminent un niveau de falaise irrégulière atteignant une cinquantaine de mètres de hauteur au droit du village, dominant une pente couverte d'éboulis orientée au Nord. L'orientation des principales familles de discontinuités détermine un découpage vertical suivant la direction de la schistosité (sensiblement N.-S.) perpendiculairement à la pente entraînant la formation d'éperons qui évoluent par écroulements successifs. L'éboulement survenu au début de l'hiver 1975, provenait de la face avant d'un éperon bien marqué dans la topographie. La distance entre la zone d'écroulement et les bâtiments les plus proches du village de SaintJean est de $350 \mathrm{~m}$ environ pour une dénivelée de $250 \mathrm{~m}$. La pente du versant qui est de 35 à $40^{\circ}$ environ à la partie supérieure dans les éboulis s'atténue à la partie inférieure. Un chemin forestier s'élève en lacets dans la pente en amont du village.

Fig. 1. - Partie supérieure de I'éboulement. La zone d'arrachement est nettement visible sur la partie avant d'un piton rocheux.

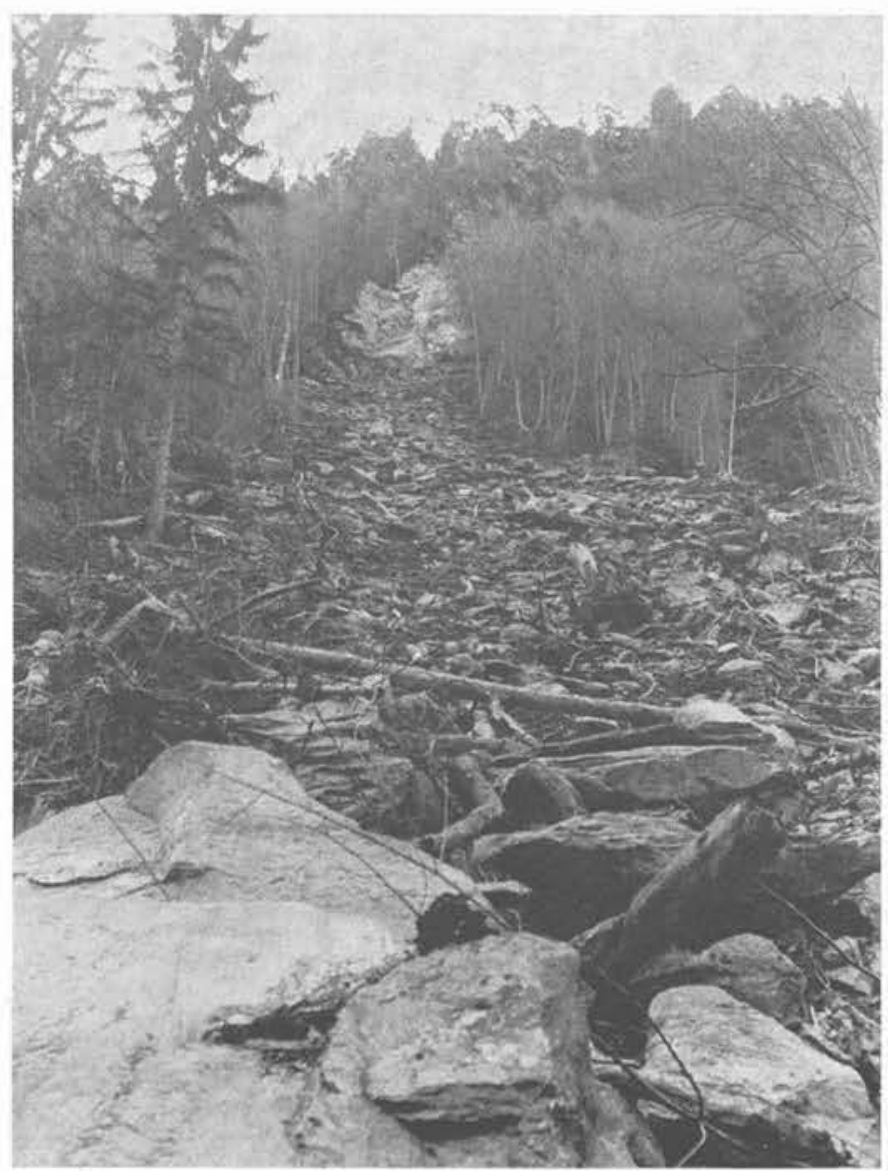




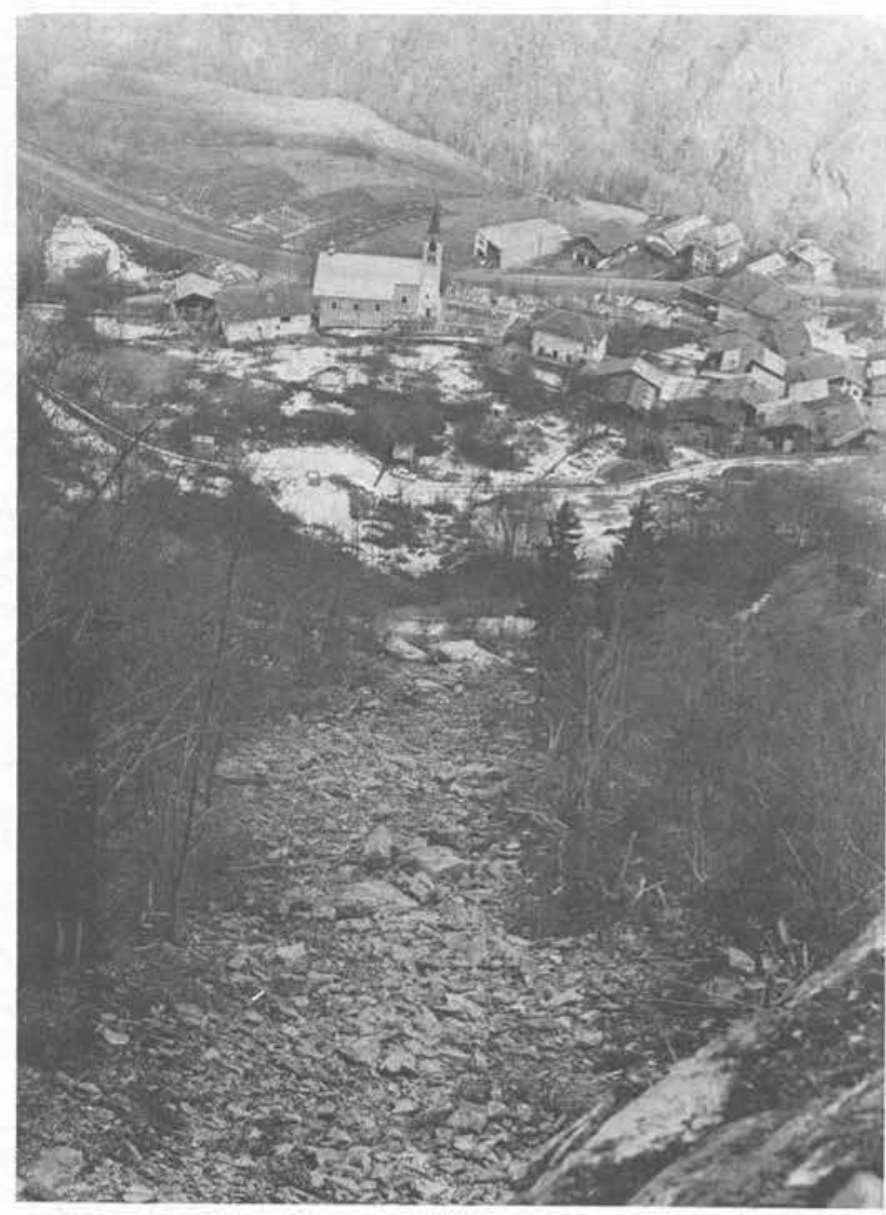

Fig. 2. - Vue d'ensemble du corps de l'éboulement et du village situé dans la trajectoire.

Fig. 3, - Chemin forestier recoupant la trajectoire de l'éboulement. Une partie des éboulis (dont de gros blocs) a été arrêtée par la piste. bâtiments sont particulièrement exposés (mairie, école, église). Les observations effectuées sur le sîte ont permis de dégager un certain nombre d'éléments importants :

- la présence du chemin forestier qui recoupe à plusieurs reprises la trajectoire de l'éboulement à joué un rôle essentiel dans la limitation de la propagation des matériaux éboulés (fig 3). Bien que la largeur de la piste ait été modeste (généralement inférieure à $3 \mathrm{~m}$ ), les lacets successifs ont contribué au stockage d'une partie des matériaux éboulés et à l'arrêt de plus gros blocs dont certains atteignaient un volume d'une dizaine de $\mathrm{m}^{3}$;

- la couverture forestière du versant n'assure aucune protection efficace contre les éboulements en masse. L'observation montre que les arbres les plus importants dont le diamètre atteignait 0.50 à $0.60 \mathrm{~m}$ ont été sectionnés à des hauteurs variables et parfois à leur base ;

- les zones de stockage doivent présenter un volume ou un nombre suffisants. Leur comblement par les matériaux éboulés leur faisant perdre leur efficacité, des travaux d'entretien sont nécessaires pour maintenir la capacité de stockage ;

- l'observation de l'état de la falaise dans la zone de l'éboulement a montré l'existence de fissures importantes délimitant un compartiment rocheux dont le volume estimé était de plusieurs milliers de mètres cubes, et dont la stabilité paraissait douteuse.

\subsection{Mesures de protection}

En conséquence, les, mesures de protection mises en œuvre ont porté sur deux points :

- Etablissement d'une succession de fossés de protection en mettant à profit les lacets successifs du
Les observations qui ont été effectuées après l'éboulement soulignent l'importance du risque auquel se trouve exposé le village. Le village de Saint-Jean se trouve placé dans la trajectoire directe des éboulements provenant de cette partie de la falaise (fig. 2) et certains

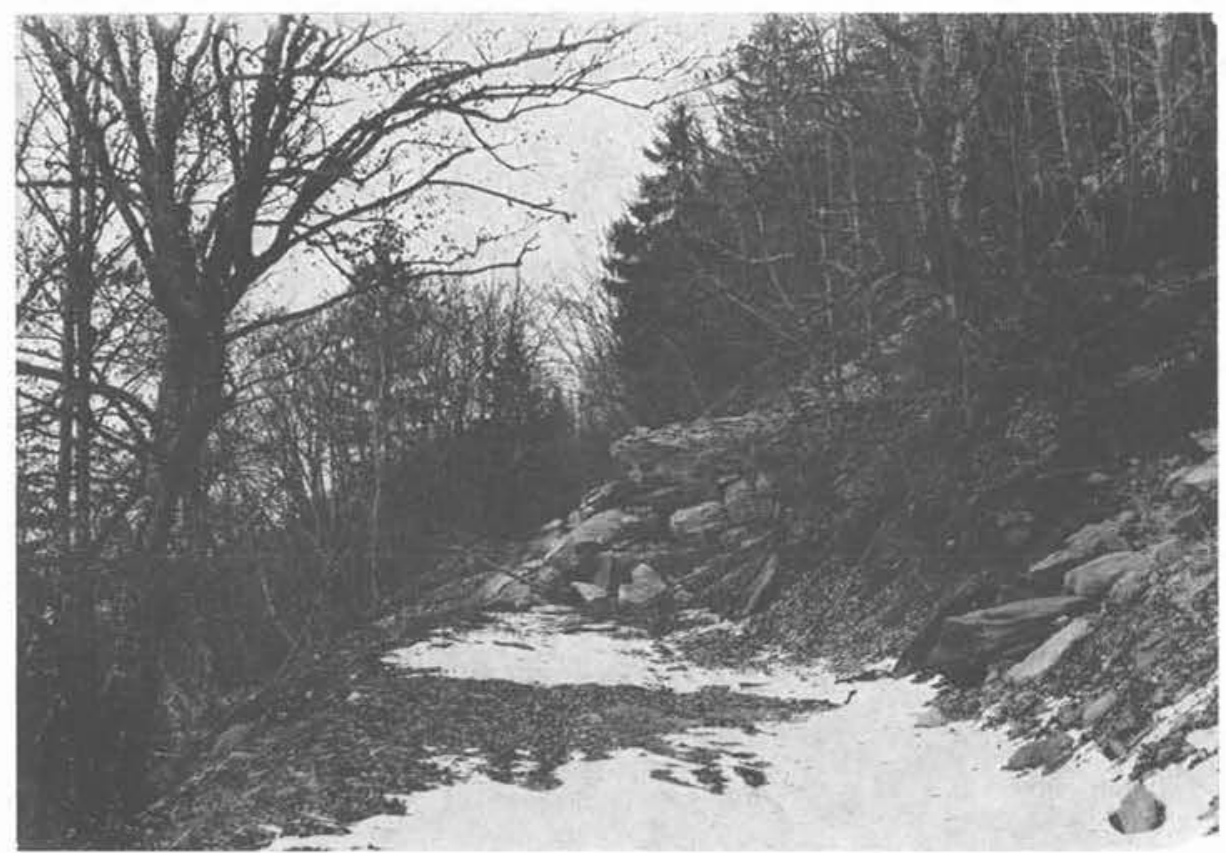

chemin forestier. Les conditions topographiques ne permettaient pas d'établir une fosse de réception de capacité suffisante à la partie supérieure de la pente. La solution adoptée a consisté à améliorer la capacité du stockage du chemin forestier, dont 


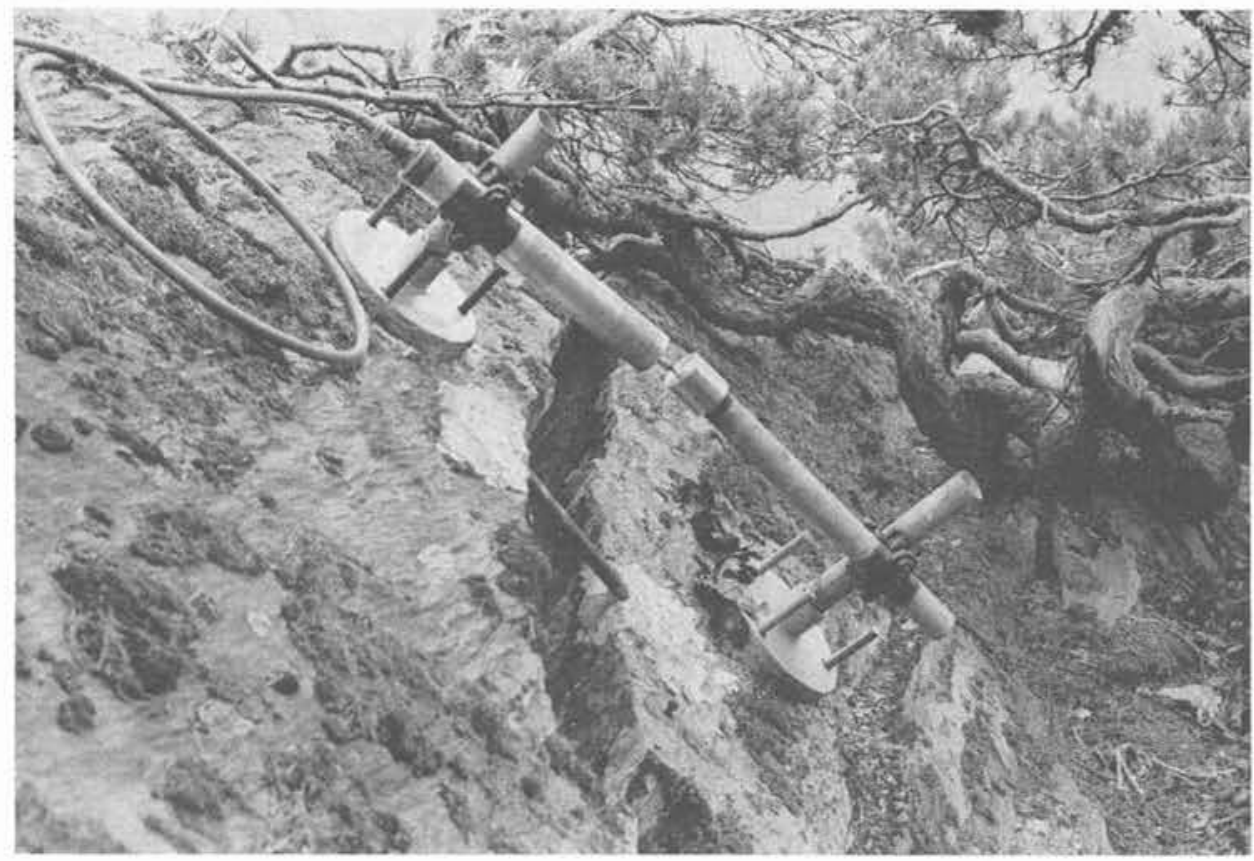

Fig. 4. - Capteur de déplacement (Tedep) en place sur une fissure ouverte délimitant un compartiment ins table de la falaise.

l'efficacité a été démontrée, par la mise en place d'une banquette en remblai du côté aval et un élargissement modéré de la piste côté amont. Des dispositions particulières ont été prises pour assurer une évacuation des eaux de ruissellement.

- Mise sous surveillance de la partie de l'éperon rocheux dont la stabilité paraissait douteuse. Un dispositif de télésurveillance (système TEDEP mis au point par les laboratoires des Ponts et Chaussées) a été mis en place pour détecter les mouvements éventuels du compartiment observé par rapport à la partie arrière de l'éperon. L'ensemble est constitué par deux capteurs de déplacements mis en place à la partie supérieure et à la partie inférieure de la fissure principale qui délimite le compartiment à surveiller (fig. 4). Les indications des capteurs sont transmises à distance jusqu'à un terminal de mesure installé dans le bâtiment de la mairie (fig. 5). Les informations des capteurs sont transmises par câble sur une distance de $600 \mathrm{~m}$ environ entre la falaise et le poste de surveillance.

L'installation a été mise en service au printemps 1975 et fonctionne régulièrement depuis cette date. Compte tenu des observations effectuées, la fréquence de mesure adoptée est quotidienne.

Les résultats des mesures effectuées depuis un peu moins de deux ans mettent en évidence l'existence de mouvements sensibles au niveau de la fissure observée (fig. 6).

Les mouvements observés montrent l'existence :

- D'une composante cyclique annuelle dont l'amplitude est de l'ordre de 3 à $4 \mathrm{~mm}$ liée aux variations climatiques. Le mouvement se fait dans le sens de la fermeture en été et de l'ouverture en hiver.

- D'une évolution à plus long terme qui se traduit par une ouverture progressive de la fissure. L'ouverture observée est de 1.5 à $2 \mathrm{~mm} / \mathrm{an}$.

L'analyse des résultats des mesures effectuées au cours de la période écoulée fournit une indication extrêmement précieuse pour la détermination du comportement des masses rocheuses instables et permet de préciser leur évolution. Sur le plan de la surveillance, ces premières indications permettent d'étalonner

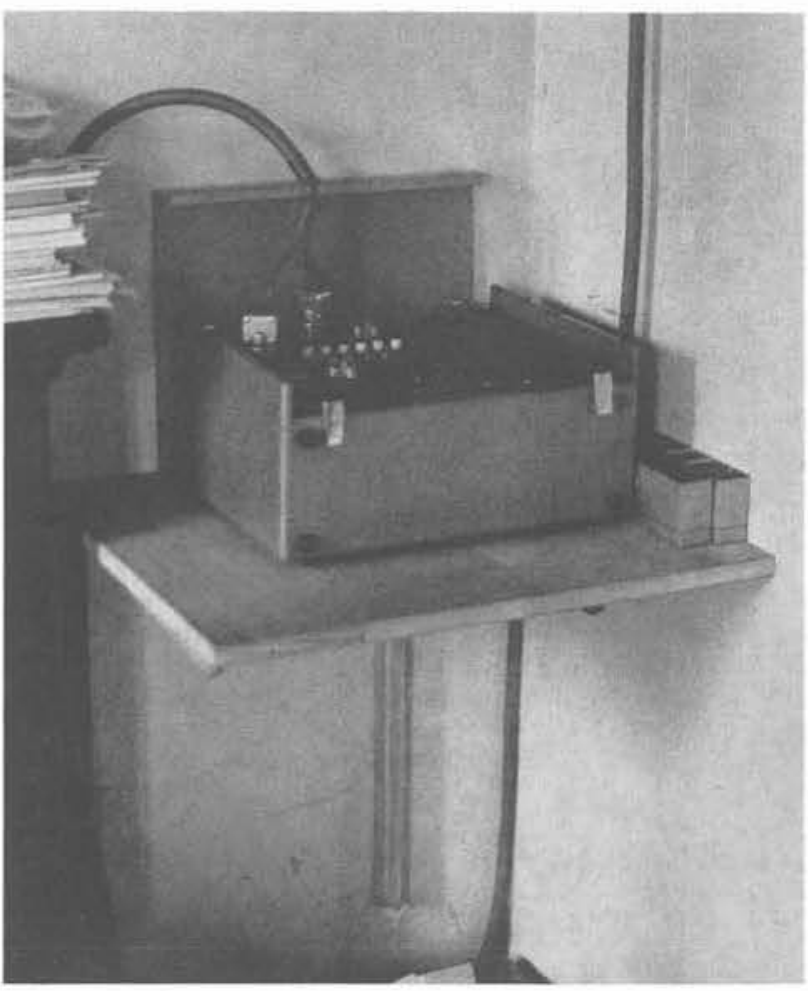

Fig. 5. - Terminal de mesure installé dans un bâtiment du village.

les observations et permettent par voie de conséquence de mettre en évidence toute évolution qui s'écarterait du comportement habituel et qui pourrait traduire une aggravation des conditions de stabilité. Les mesures de surveillance constituent un élément indispensable de la protection et sont poursuivies de manière active.

D'une manière plus générale, l'interprétation des mesures reste toujours un problème délicat. Une période d'initialisation des observations est toujours nécessaire pour permettre une analyse des différents facteurs qui déterminent le comportement des masses observées. 
Fig. 6. - Evolution de l'ouverture des fissures principales délimitant un compartiment ins table de la falaise.
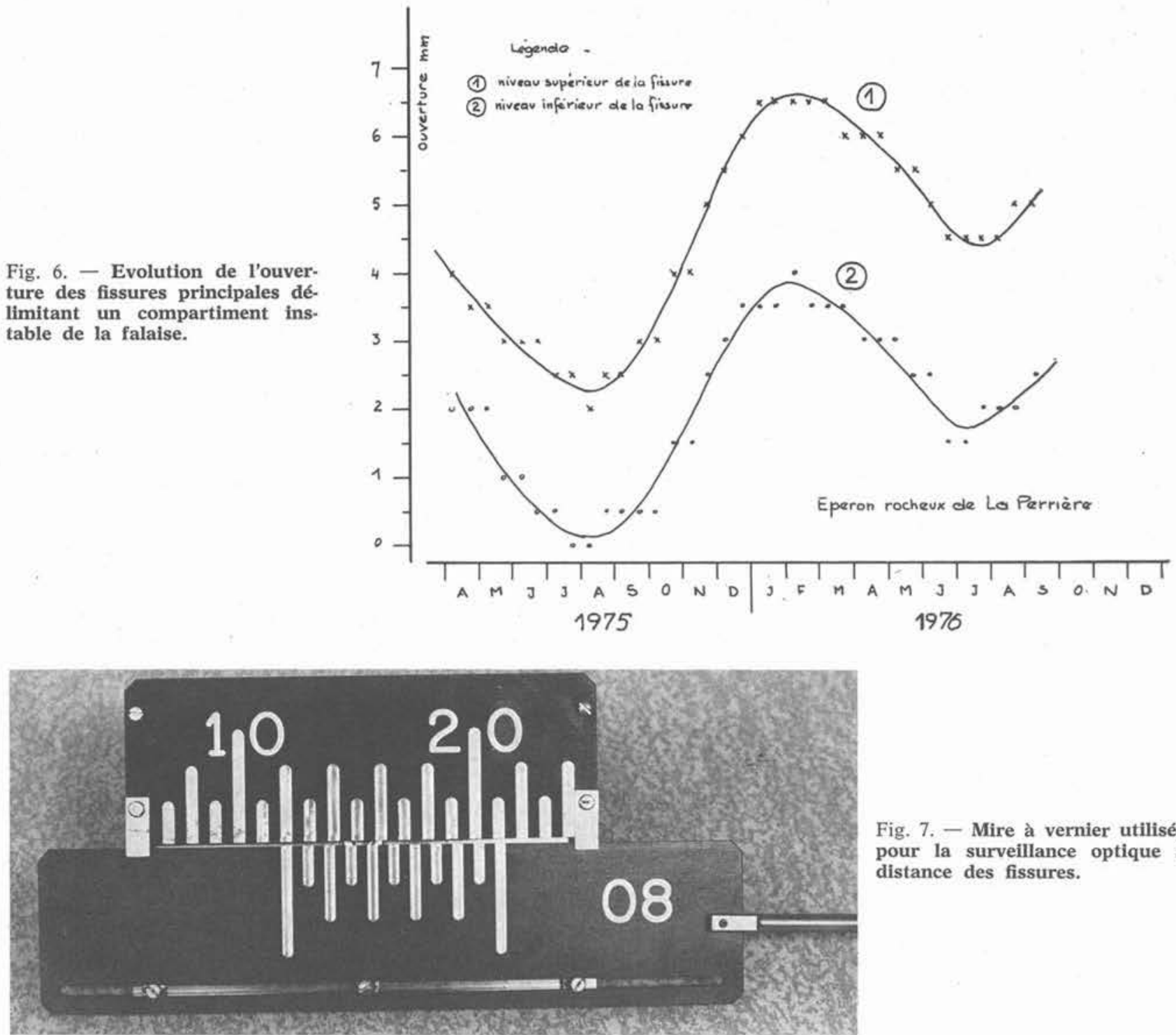

Fig. 7. - Mire à vernier utilisée pour la surveillance optique à distance des fissures.

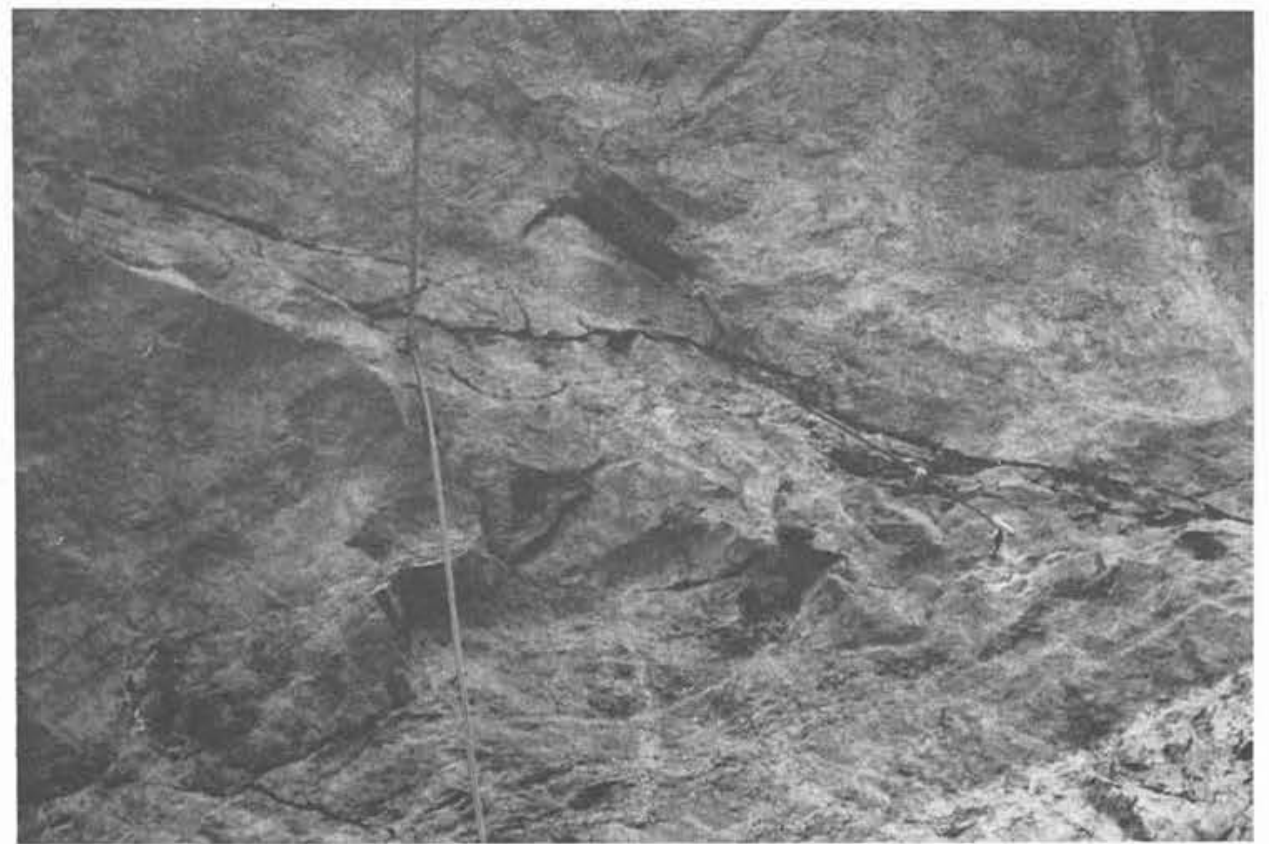

Fig. 8. - Mire à vernier en place sur une fissure (mesure du mouvement de cisaillement des épontes). 
Les observations ne peuvent être interprétées isolement mais en fonction de la connaissance que l'on a du comportement antérieur. Cette connaissance s'améliore au fur et à mesure que s'accumulent les observations. L'interprétation s'appuie essentiellement sur l'analyse de la cinématique des phénomènes (amplitude, vitesse, accélération...).

La multiplication des observations relatives au comportement des masses rocheuses instables est un élément essentiel pour l'amélioration des connaissances et de l'efficacité dans le domaine de la prévention et de la protection. Les possibilités d'instrumentation sont généralement limitées par des considérations économiques. Divers dispositifs de mesure ont été mis au point pour permettre néanmoins un développement des mesures in situ. On peut citer un système de mires à vernier (fig. 7 et 8 ) mis au point par les laboratoires des Ponts et Chaussées utilisé sur plusieurs sites. Ces appareillages d'une mise en œuvre peu onéreuse permettent la mesure à distance du mouvement des fissures ou du mouvement relatif de compartiments rocheux (lecture jusqu'à $200 \mathrm{~m}$, précision $\pm 1 \mathrm{~mm}$ ).

\section{CONCLUSIONS}

La protection de zones exposées à des éboulements rocheux pose souvent de difficiles problèmes par la difficulté d'une appréciation objective des risques et l'importance des moyens nécessaires. La mise en œuvre de parades efficaces succite une analyse des conditions de l'instabilité des masses concernées, une estimation des volumes en cause et une prévision des mécanismes qui paraissent les plus probables.

La surveillance constitue un élément indispensable de la protection. Seules des mesures précises permettent de déterminer les caractéristiques de l'évolution des phénomènes et de les saisir dans leur aspect dynamique. Les mesures de surveillance permettent une analyse du comportement des masses instables et la recherche de signes prémonitoires précoces permettant de définir des critères suffisamment fiables auxquels on puisse associer la mise en œuvre de mesure de sauvegarde. De grands progrès restent encore à faire dans ce domaine. 